- Estudi -

\title{
ENERGÍAS RENOVABLES Y EFICIENCIA ENERGÉTICA EN EL ÁMBITO LOCAL: DISPOSITIVOS DE INTERVENCIÓN AL ALCANCE DE LOS ENTES LOCALES
}

\author{
Manuela MORA RUIZ \\ Profesora Contratada Doctora de Derecho Administrativo \\ Departamento de Derecho Público. Universidad de Huelva ${ }^{1}$ \\ manuela@uhu.es
}

Recibido: 28 de febrero de 2012 / Aceptado: 4 de mayo de 2012

RESUMEN: Las energías renovables y el ahorro y eficiencia energética constituyen dos aspectos fundamentales en la lucha contra el cambio climático. En este contexto, las Administraciones Públicas asumen distintas funciones que tienen proyección directa en instrumentos jurídicos específicos en la ordenación de este sector. En este sentido, las Entidades Locales parecen tener una posición limitada en el impulso de las energías renovables y la eficiencia energética, que contrasta con el espacio y competencias asumibles por las mismas al amparo del concepto de sostenibilidad urbana. Concretar este aspecto constituye el objeto fundamental de este Trabajo. Se exponen, así, los elementos generales de ordenación de las energías renovables y la capacidad de las entidades locales para poder desarrollar la función pública de tutela ambiental en un sector tan complejo como el energético, en el que la producción de estas energías, junto a las políticas de ahorro y eficiencia energética, no solo se articulan a partir de las exigencias de protección del medio ambiente, sino también teniendo en cuenta las especialidades de un sector estratégico.

\footnotetext{
${ }^{1}$ Este artículo trae causa de la participación en el Seminario "Acción Administrativa y Sostenibilidad" organizado por el profesor A. FORTES MARTÍN, de la Universidad Carlos III, en noviembre de 2011, a quien agradezco su invitación, y se realiza en el marco del Proyecto del Estado DER2010-16543, sobre Procedimiento Administrativo: simplificación, mejora de eficacia y garantías de los ciudadanos.
} 
ABSTRACT: Renewable energies and efficiency are the means to fight against the Climate Change. From this point of view, the Administration has new functions and, therefore, new instruments to implement in this area. In this context, Local Governments seem to play a limited role concerning these energies and the efficiency, but their real position is quite different because of the concept of urban sostenibility. This Paper aims to show which legal instruments can be used at the local level of government to promote the renewable energies and efficiency. In doing so, this Paper shows the legal regulation of this field, in order to make evident that is a very difficult question, because of the relationship between the environmental remarks of the renewable energies and efficiency an the energies, as a regulated field of the Administrative Law.

PALABRAS CLAVES: Energías renovables; eficiencia energética; medio ambiente urbano sostenible; planificación; intervención.

KEY WORDS: Renewable energies; energetic efficiency; urban sostenibility; planning; management.

Sumario: I. Una cuestión previa: Entes Locales, medio ambiente y desarrollo sostenible. II. Energías renovables y ahorro y eficiencia energética: las peculiaridades de la ordenación jurídico-administrativa del sector. Proyección en el nivel local. III. Dispositivos de actuación y/o intervención de los Entes Locales en materia de energías renovables y eficiencia energética. IV. Consideraciones finales. V. Bibliografía citada.

\section{UNA CUESTIÓN PREVIA: ENTES LOCALES, MEDIO AMBIENTE Y DESARROLLO SOSTENIBLE}

Las energías renovables y la eficiencia energética constituyen un ámbito material de difícil comprensión y estructuración, en lo que a su ordenación jurídica se refiere, porque en él confluyen consideraciones ambientales, ligadas estrechamente a las políticas de lucha contra el cambio climático, junto a consideraciones económicas vinculadas a las necesidades de abastecimiento energético de las sociedades: a nuestro juicio, estamos ante un sector de gran importancia desde la perspectiva del Derecho 
Administrativo, tanto por los instrumentos jurídicos que contribuyen a la ordenación del mismo como por la peculiar posición de las Administraciones en la aplicación de dicha ordenación, ya que han de tener muy presente la necesidad de equilibrio entre las exigencias ambientales y las económicas a las que antes nos referíamos.

Desde esta perspectiva, el presente Trabajo pretende poner de manifiesto la especificidad de la intervención administrativa en un sector tan peculiar como el de las energías renovables y la eficiencia energética cuando se desenvuelve en el nivel local, ya que el carácter global de las estrategias de lucha contra el cambio climático, junto con la trascendencia de las decisiones sobre política energética, parecen situarse en un nivel diferente al de las Administraciones Locales, lo que reduce en gran medida las posibilidades de participación de éstas en el ámbito que nos ocupa. A nuestro juicio, este es un planteamiento equívoco, que precisa, para su superación, la determinación del ámbito exacto que las Administraciones Locales pueden ocupar en la generalización del recurso a las energías renovables y en la puesta en marcha de políticas de ahorro y eficiencia energética, para poner de manifiesto la relevancia de los principios ordenadores de las relaciones interadministrativas, puesto que las fórmulas de intervención que se adopten en el ámbito local y las decisiones que se lleven a término trascienden este ámbito y contribuyen a concretar el régimen jurídico de estas energías $y$, en su caso, de la eficiencia energética.

En este sentido, el punto de partida de las afirmaciones anteriores se encuentra en la comprensión del principio de desarrollo sostenible, que corresponde a municipios y provincias puesto que la ciudad es, prima facie, "insostenible", para precisar, entonces, la concreción del aludido principio en este espacio físico:

Por un lado, está clara la implicación de las Entidades Locales en la tutela ambiental, al amparo tanto de la función pública del art. 45.2 CE como por las exigencias del principio de subsidiariedad de la acción pública, en el sentido de reconocer la idoneidad de la instancia descentralizada para la realización de un interés general tan fundamental

\footnotetext{
${ }^{2}$ Así lo pone de manifiesto TAMAMES, R., tal y como señala LORA-TAMAYO, M.: "La integración normativa de la dimensión medioambiental en el medio urbano. El desarrollo urbano sostenible y su implementación”, en PARADA VÁZQUEZ, R., FUENTETAJA PASTOR, A., Reforma y retos de la Administración local, Ed. Marcial Pons, Madrid, 2007, pág. 324: La ciudad “[...] sustituye recursos no renovables (la tierra fértil) por asfalto y hormigón y desborda de manera desmesurada la capacidad de carga del territorio, y utiliza recursos de territorios distantes; por esta razón, el proceso de urbanización es visto desde una perspectiva ambiental como insostenible".
} 
como la tutela del medio ambiente ${ }^{3}$. Por otro lado, sin embargo, la realidad es bien diferente, y ello por la confluencia de diversos factores que, en definitiva, colocan en un plano secundario y limitado la acción local administrativa sobre el medio ambiente. Así, baste pensar en las mínimas consideraciones que el Borrador del Plan de Energías Renovables 2011-2020 dedica a los Entes Locales ${ }^{4}$; en las dificultades para participar en los procedimientos de elaboración de normas en función del "grupo normativo" del que se trate $-\mathrm{y}$, por tanto, de la presión que ejercen grupos de poder de carácter privado— ${ }^{5}$; o en la dificultad técnica de las materias que se regulan en las normas ambientales, lo cual permite reconocer una cierta tendencia a que los niveles superiores con potestad normativa asuman el grueso de las regulaciones, desplazándose el eje de toma de decisiones hacia instancias superiores ${ }^{6}$.

En este escenario, como señala E. Alonso ${ }^{7}$, los Entes Locales deben poder incrementar el nivel de protección del bien jurídico que nos ocupa por la vía de diseñar su propia política ambiental de carácter local, y, en nuestra opinión, esta posibilidad viene de la mano de la noción de desarrollo sostenible, que permite reconocer un ámbito de actuación propio de estas entidades, legitimador de su intervención en materia de energías renovables y eficiencia energética. Para ello, el desarrollo sostenible ha de conceptualizarse, en primer término, conforme al concepto comunitario ${ }^{8}$ de este

\footnotetext{
${ }^{3}$ ALONSO GARCÍA, E., en "La gestión del medio ambiente por las entidades locales", en MUÑOZ MACHADO, S. (dir.), Tratado de Derecho Municipal, Tomo II, Iustel, Madrid, 2011, pág. 2.456: el autor reconoce que las competencias clásicas ambientales de las Entidades Locales han sido de "arbitraje", en torno a las relaciones de vecindad, para evolucionar hacia las competencias de control, en el marco del RAMINP, y después hacia un mayor protagonismo de instancias inferiores, no sólo en la implementación sino también en el diseño de políticas ambientales.

${ }^{4}$ Redacción de 26 de julio de 2011. De hecho, este es el contenido de la alegación que realiza la Federación Española de Municipios y Provincias en el trámite de información pública: "Se obvia el ámbito local o municipal en la enumeración de normativa que afecta a la regulación de las ER, obviando las Ordenanzas y Reglamentos que pueden desarrollar las Corporaciones Locales y no teniendo en cuenta el destacado papel que desempeñan los Ayuntamientos en la elaboración de las Ordenanzas relativas a la regulación de la energía solar". El plan definitivo fue aprobado por Acuerdo del Consejo de Ministros de 11 de noviembre 2011, y puede consultarse en el siguiente enlace: $<$ http://www.idae.es/index.php/mod.documentos/mem.descarga?file=/documentos_11227_PER_20112020_def_93c624ab.pdf $>$.

${ }^{5}$ Véase ALONSO GARCÍA, "La gestión...”, op. cit., pág. 2.457. Desde esta perspectiva, piénsese en la relevancia económica de las energías renovables como parte del sector energético.

${ }^{6}$ Véase ALONSO GARCÍA, “La gestión...”, op. cit., pág. 2.503.

${ }^{7}$ Véase op. cit., pág. 2.456.

8 Véase FERNÁNDEZ DE GATTA, D., "El régimen de sostenibilidad medioambiental", en Revista Jurídica de Castilla y León, 25 septiembre de 2011, pág. 169. Para el autor, el desarrollo sostenible en el ámbito europeo "[...] es un tipo de desarrollo que propugna en primer lugar la armonización entre el desarrollo económico y la protección del medio ambiente, añadiéndole el progreso social”, de forma que
} 
principio, de forma que lo económico, lo ambiental y lo social quedan engarzados; y, por otra parte, ha de concretarse en dispositivos tan específicos como las llamadas Agendas Locales 21, consideradas como el marco a partir del cual los municipios, sobre todo, están en condiciones de diseñar una estrategia de "sostenibilidad integral", pues el principio que nos ocupa ha llegado a considerarse "Título habilitante"10 a partir del cual las Administraciones Públicas han de concretar una determinada forma de actuación que les lleve a compatibilizar las aludidas exigencias de tutela ambiental con el crecimiento económico y el desarrollo social.

A nuestro juicio, lo anterior se concreta en el concepto jurídico de medio ambiente urbano sostenible, a partir del cual todas las Administraciones han de lograr los objetivos del art. 107 de la Ley 2/2011, de 4 de marzo, de Economía Sostenible ${ }^{11}$, relativo a los "fines comunes de las políticas públicas para un medio urbano sostenible"-, en cuya virtud los poderes públicos, de acuerdo con los principios de cohesión territorial y social, eficiencia energética y complejidad funcional, formularán y desarrollarán las políticas de su respectiva competencia al servicio de este medio ambiente urbano sostenible, lo que implica, entre otros aspectos, un entorno urbano en el que:

“a) Posibilitar el uso residencial en viviendas constitutivas de domicilio habitual en un contexto urbano seguro, salubre y adecuado, libre de ruidos y otras inmisiones contaminantes que excedan de los límites legalmente permitidos en cada momento y provisto del equipamiento, los servicios, los materiales y productos que eliminen

el resultado puede considerarse armónico entre los tres pilares. En la misma línea, SANZ LARRUGA, J., "Sostenibilidad ambiental y Derecho Administrativo: ¿nuevo remedio ante la crisis económica o una exigencia constitucional? A propósito de la nueva Ley de Economía Sostenible", en BLASCO ESTEVE, A., (coord.), Actas del VI Congreso de la Asociación Española de Profesores de Derecho Administrativo, INAP, Madrid, 2011, pág. 442, al señalar que, para el Derecho Administrativo, "la sostenibilidad ambiental no deja de ser una aplicación de la eficacia administrativa desde la perspectiva del criterio de la eficiencia como principio de utilización racional de los recursos".

9 En este sentido, las Agendas 21 surgen en la Cumbre de Río para crear el marco en el que las Comunidades Locales puedan alcanzar modelos de sostenibilidad para el s. XXI, concretándose a partir de la Cumbre de Alborg de 1994. En todo caso, su interés estriba en que legitiman a los entes locales para determinar una "política ambiental integral" a partir del análisis previo de su situación, lo cual puede tener especial trascendencia en la aprobación de "Ordenanzas Ambientales de Sostenibilidad": ALONSO GARCÍA, "La gestión...”, op. cit. pág. 1.525.

${ }^{10}$ Véase ibídem...op. cit. pág. 171.

${ }^{11}$ Para un estudio más completo sobre la significación de esta norma en materia de energías renovables entre otros aspectos de renovación de este sector, véase GONZÁLEZ RÍOS, I., Régimen jurídicoadministrativo de las energías renovables y de la eficiencia energética, Ed. Aranzadi, Cizur-Menor, Navarra, 2011, in totum. 
o, en todo caso, minimicen, por aplicación de la mejor tecnología disponible en el mercado a precio razonable, las emisiones contaminantes, el consumo de agua, energía y la producción de residuos, y mejoren su gestión.

b) Mejorar la calidad ambiental y la funcionalidad de las dotaciones, infraestructuras y espacios públicos al servicio de todos los ciudadanos.

c) Fomentar unos servicios generales, incluidos los que aseguren el acceso universal a las nuevas tecnologías, más eficientes económica y ambientalmente en la gestión del agua, la energía y los residuos.

d) Favorecer, por contar con las infraestructuras, dotaciones y servicios precisos, la localización de actividades de contenido económico generadoras de empleo estable y sostenibles medioambientalmente, especialmente las que faciliten el desarrollo de la investigación científica y de nuevas tecnologías.

e) Garantizar el acceso equitativo de los ciudadanos a las dotaciones y los servicios y su movilidad, potenciando al efecto los medios de transporte, especialmente los de uso público, más eficientes energéticamente y menos contaminantes.

f) Integrar en el tejido urbano cuantos usos resulten compatibles con la función residencial, contribuyendo al equilibrio de ciudades y núcleos residenciales, para lo que se favorecerá la aproximación de servicios y dotaciones a la comunidad residente, evitando desplazamientos innecesarios.

g) Fomentar el uso de materiales, productos y tecnologías limpias que reduzcan las emisiones contaminantes del sector de la construcción".

El efecto inmediato de un precepto como el que acabamos de referir no es otro que el de concretar la noción de medio ambiente urbano sostenible, en el sentido de que será aquel que no sólo va a nutrirse de políticas ambientales en sentido estricto, sino que habrá de incorporar las exigencias de desarrollo económico y cohesión social que precise la comunidad $^{12}$, jugando, en lo que a este Trabajo interesa, un papel fundamental el impulso de las energías renovables y la eficiencia energética. La cuestión será, entonces, qué instrumentos jurídicos están al alcance de los Entes Locales para lograr un desarrollo sostenible en los términos expuestos, y ello con el apoyo de planteamientos más generales como los que se recogen en la Estrategia Europa 20-20-20 y la

12 LORA-TAMAYO VALLVÉ, en "La integración...." op. cit., pág. 325, emplea el concepto de "desarrollo urbano sostenible" para referirse a "un supraconcepto integrador y (que) se configura científicamente como la compleja interacción trilateral entre el entorno económico, el social y el ecológico". 
aprobación del llamado Pacto de los Alcaldes, con clara repercusión sobre el Gobierno Local $^{13}$.

El objetivo, así pues, de este Trabajo será el análisis del espacio que corresponde a las Administraciones Locales en el impulso de las energías renovables y el ahorro y la eficiencia energética, a partir de un punto de salida indiscutible como el que representa la noción de medio ambiente urbano sostenible, en cuya virtud la Entidad Local queda claramente legitimada para articular una política propia sobre este sector en la que concretar su grado de intervención y/o los instrumentos jurídicos precisos al efecto. A ello dedicaremos los siguientes epígrafes, donde pondremos de manifiesto que la dificultad del estudio estriba, sobre todo, en la complejidad de reconducir a unidad la actuación de las Administraciones Locales, cuyas iniciativas, en un marco poco preciso en lo que a sus competencias sobre medio ambiente se refiere, son, en su mayoría, dispares.

\section{ENERGÍAS RENOVABLES Y AHORRO Y EFICIENCIA ENERGÉTICA:} LAS PECULIARIDADES DE LA ORDENACIÓN JURÍDICOADMINISTRATIVA DEL SECTOR. PROYECCIÓN EN EL NIVEL LOCAL

Admitida la relación entre la Administración Local y las energías renovables, así como con el ahorro y la eficiencia energética, sobre la base de la idea del desarrollo urbano sostenible, el paso previo a la consideración de los instrumentos jurídicos concretos a través de los cuales conseguir los objetivos de generalización de estas energías y la reducción de consumos energéticos precisa dejar sentadas algunas premisas sobre la concreta ordenación jurídico-administrativa de esta materia, en tanto en cuanto ello nos

\footnotetext{
${ }^{13}$ El Pacto de los Alcaldes es una iniciativa de la Comisión Europea para articular una red de alcaldes a través de la cual sea posible el intercambio permanente de información para la puesta en marcha de buenas prácticas, con el objetivo de mejorar la eficiencia energética del entorno urbano. Así se recoge en el "Análisis de los compromisos derivados de la adhesión al Pacto de Alcaldes", de 19 de noviembre de 2008, que encarga la Federación Española de Municipios y Provincias (en adelante, FEMP). El Pacto se pone en marcha en la Sesión Plenaria de la VII Conferencia Anual ManagEnergy, que tuvo lugar el 29 de enero de 2008, como parte de la II Semana de la Energía Sostenible de la UE. En el Documento elaborado por la FEMP, las líneas estratégicas de actuación se relacionarían con la articulación de una política municipal y la concreción del modelo de ciudad correspondiente, la movilidad sostenible, la potenciación de las energías renovables, la actuación en sectores concretos-industrial, agricultura, pesca, ganaderíao la configuración de los Ayuntamientos como líderes de opinión o creadores de tendencias. Es evidente, en nuestra opinión, que consideraciones como esta última sitúan a las Administraciones en un plano muy diferente al de la actividad de limitación y de fomento que tradicionalmente se ha aceptado: las funciones de las Administraciones están en plena transformación.
} 
permitirá conocer la medida exacta de intervención de los Entes Locales en la materia que nos ocupa. En este sentido, la implicación de estas Administraciones en energías renovables y eficiencia energética depende, en esencia, de las competencias que el ordenamiento reconoce a las mismas sobre medio ambiente, con carácter general, y sobre energías renovables y eficiencia energética en particular ${ }^{14}$. En todo caso, cualquiera que sea el enfoque que adoptemos llegamos a idéntica conclusión, esto es, la limitada capacidad de actuación de la que disponen los Entes Locales para actuar en este ámbito, por no existir una regulación suficientemente precisa en este sentido; antes al contrario, las energías renovables y la eficiencia energética son objeto de una regulación sui generis, que combina normas en sentido estricto con una planificación de carácter indicativa $^{15}$, a través de la cual se fijan objetivos, plazos e instrumentos con diferente alcance vinculante para Administraciones y sujetos privados, y en la que confluyen normas estatales y autonómicas que, lejos de presentar un escenario jurídico claro, dan lugar a un mosaico normativo de difícil aprehensión y aplicación, en el que los Entes Locales ocupan un espacio mínimo ${ }^{16}$.

La cuestión es especialmente delicada en la actualidad, puesto que ahora tenemos un nuevo escenario, establecido por el Plan de Energías Renovables 2011-2020 ${ }^{17}$, el Plan

${ }^{14}$ En este sentido, téngase en cuenta la diferenciación que establece M. J. LÓPEZ SAKO entre la actividad local sobre las energías renovables, sea en régimen de concurrencia, sea a través de la construcción de servicios esenciales, y el ejercicio de competencias locales vinculadas a este sector, tanto en el ámbito general de las potestades administrativas del art. 4.1 Ley 7/1987, Reguladora de las Bases del Régimen Local, y en el más preciso de atribución de competencias de los arts. 25 y 26 de la misma norma. A nuestro juicio, esta diferenciación pone de manifiesto las dificultades a la hora de precisar el fundamento de la intervención de las Entidades Locales en materia de energías renovables y eficiencia energética, convirtiéndose en un obstáculo para el efectivo desenvolvimiento de una política local en este ámbito, con objetivos específicos e instrumentos jurídicos propios para la consecución de dichos objetivos. En todo caso, véase LÓPEZ SAKO, M. J. "Competencias municipales en materia de energías renovables”, en VERA JURADO, D. (coord.), Competencias ambientales de las Entidades Locales de Andalucía, CEMCI, Granada, 2009, págs. 466 a 468.

${ }^{15}$ A modo de ejemplo, el art. 5 de la Ley 50/1997, de 27 de noviembre, del Sector Eléctrico, establece el carácter vinculante de la planificación estatal relativa a las instalaciones de transporte energético, condicionando, así pues, la planificación territorial y urbanística. El precepto evidencia el limitado espacio que las Corporaciones Locales tienen en materia energética, y, por extensión, en relación con el recurso a fuentes renovables de energía.

${ }^{16}$ Sobre el peso de la normativa estatal y autonómica en la materia que nos ocupa, véase MORA RUIZ, M., "La ordenación jurídico-administrativa de las energías renovables: revisión en el marco de la Directiva 2009/28/CE, del Parlamento Europeo y del Consejo, de 23 de abril, de fomento de las energías renovables, en Revista de Derecho Urbanístico y Medio Ambiente, núm. 257, 2010, págs. 172 y ss. En sentido similar, de la misma autora véase "Los condicionantes ambientales de las energías renovables: el ejemplo de la energía eólica en el Derecho Español", en Actualidad Jurídica Ambiental, 30 de septiembre de 2010 (en <www.actualidadjuridicaambiental.com>), págs. 6 a 14.

${ }^{17}$ El plan definitivo fue aprobado por Acuerdo del Consejo de Ministros de 11 de noviembre 2011, y puede consultarse en el siguiente enlace: 
de Ahorro y Eficiencia Energética ${ }^{18}$ — con el mismo horizonte temporal- y la Directiva 28/2009/CE, de 23 de abril, del Consejo y el Parlamento Europeo, sobre el fomento de las energías renovables, que crean un marco jurídico de referencia en el que los sistemas de apoyo se convierten en la pieza clave del desarrollo de estas energías ${ }^{19}$, debiéndose procurar la aplicación de los mismos por todas las Administraciones.

Así, desde una perspectiva general no puede dudarse de la competencia de los Entes Locales en medio ambiente, en los términos ya sabidos de los arts. $25.1^{20}$ y $\left.25.2 . f\right)^{21}$, así como el art. 28 de la $\mathrm{LRBRL}^{22}$, en el sentido de reconocer una cierta competencia general para poder articular, en el marco del Derecho estatal y autonómico, una política local propia sobre medio ambiente, lo que debe ser comprensivo del fomento de las energías renovables y el ahorro y eficiencia energética ${ }^{23}$. Junto a ello, tampoco puede obviarse la competencia de los municipios en materia de urbanismo, en suministro de agua y alumbrado; o en tratamiento de aguas e, incluso, en transporte público de viajeros o en la ordenación del tráfico de vehículos — art. 25.2. d), 1) ${ }^{24}$, 11) o b) ninguna de estas competencias alude específicamente a la materia que nos ocupa, pero

$<$ http://www.idae.es/index.php/mod.documentos/mem.descarga?file=/documentos_11227_PER_20112020 def 93c624ab.pdf $>$, visitado el 31 de mayo 2012.

${ }^{18}$ Este Plan de Acción de Ahorro y Eficiencia Energética 2011-2020 ha sido aprobado por Acuerdo de Consejo de Ministros de 29 de julio de 2011 y da cumplimiento a lo exigido por la Directiva 2006/32/CE, del Parlamento Europeo y del Consejo, de 5 de abril de 2006, sobre la eficiencia del uso final de la energía y los servicios energéticos (DOUE de 27 de abril de 2006). El Plan puede localizarse en $<$ http://www.idae.es/index.php/mod.documentos/mem.descarga?file=/documentos_11905_PAEE_2011_2 020._A2011_57bf5075.pdf>, visitada el 17 de febrero de 2012.

${ }^{19}$ Véase LÓPEZ SAKO, M. J., Regulación y Autorización de los parques eólicos, Thomson-Civitas, Madrid, 2008, pág. 169: el autor se refiere al art. 13.3 de la Directiva de Fomento de las Energías Renovables y la previsión de otras medidas, como el uso de las energías renovables en la planificación de zonas industriales y residenciales y, en particular, de infraestructuras urbanas, lo cual debe traducirse en un mayor protagonismo de los Entes Locales. No obstante, el autor alude al carácter blando de la disposición, que puede dar lugar a que las Ordenanzas no tengan suficiente cobertura legal.

${ }^{20}$ El precepto dispone lo siguiente: "El Municipio, para la gestión de sus intereses y en el ámbito de sus competencias, puede promover toda clase de actividades y prestar cuantos servicios públicos contribuyan a satisfacer las necesidades y aspiraciones de la comunidad vecinal”.

21 “El Municipio ejercerá, en todo caso, competencias en los términos de la legislación del Estado y de las Comunidades Autónomas, en las siguientes materias:...f) Protección del medio ambiente".

22 "Los Municipios pueden realizar actividades complementarias de las propias de otras Administraciones públicas $\mathrm{y}$, en particular, las relativas a la educación, la cultura, la promoción de la mujer, la vivienda, la sanidad y la protección del medio ambiente".

${ }^{23}$ Véase supra nota al pie 13.

${ }^{24}$ En el mismo sentido, téngase en cuenta el art. 28.1.b) Real Decreto-Legislativo 781/1986, de 18 de abril, por el que se aprueba el Texto Refundido de las Disposiciones Legales vigentes en materia de Régimen Local, que dispone la competencia del Pleno para establecer el suministro eléctrico. 
es evidente que legitiman para tomar decisiones que, en los términos que veremos ahora, se vinculan a las energías renovables y la eficiencia energética.

La consecuencia inmediata de lo anterior es que debemos concretar el espacio competencial de los Entes Locales, especialmente de los municipios, sobre la base de la ordenación jurídico-administrativa a la que antes nos referíamos. De lo cual destacamos los siguientes aspectos:

En primer lugar, la regulación de las energías renovables y la eficiencia energética pueden articularse a través de normas diversas, en el sentido de que no deben coincidir necesariamente, pero suelen ir de la mano, en tanto en cuanto se vinculan a una línea estratégica de lucha contra el cambio climático, que es compartida ${ }^{25}$. En consecuencia, una y otra regulación se plantean de manera conjunta, lo que propicia que las iniciativas y medidas que se adoptan en esta materia se planteen en un nivel superior al de los Entes Locales, cuyo papel acaba diluyéndose.

No obstante lo anterior, no hay duda de que un ámbito natural para la actuación de los Entes Locales es el de los llamados sectores difusos, que se escapan a la aplicación de otros dispositivos de lucha contra el cambio climático, como el mercado de derechos de emisión de $\mathrm{CO}_{2}$ : la edificación, el transporte urbano, el sector residencial o comercial constituyen ámbitos con los que las Administraciones Locales se encuentran estrechamente relacionados $^{26}$, disponiendo, así, de una posición privilegiada para la ordenación de estos sectores desde la perspectiva ambiental, por la vía de recurrir a la multiplicidad de competencias que les corresponderían en estos ámbitos, en los términos que ya se han comentado, y al amparo de un general principio de subsidiariedad de la acción administrativa, favorecedor, a nuestro juicio, de la actuación del nivel organizativo más eficaz.

Por otro lado, las energías renovables y la eficiencia energética son objeto de regulación de manera diversa en normas y en planes que, como antes hemos señalado, son ejemplos de una planificación indicativa, vinculante para las Administraciones pero de

\footnotetext{
${ }^{25}$ Véase LÓPEZ SAKO, M. J., "Competencias municipales en materia de energías renovables" op. cit., págs. 480-481.

${ }^{26}$ CANALS I AMETLleR, D., "Las Administraciones en el cumplimiento del Protocolo de Kioto. El mercado de derechos de emisión de gases de efecto invernadero y otros instrumentos", en ESTEVE PARDO, J. (dir.), Derecho del Medio Ambiente y Administración Local, 2a Edición, Fundación Democracia y Gobierno Local, Barcelona, 2006, pág. 562: no puede obviarse la participación de los Entes
} 
forma particular, a través de la programación de las actuaciones de la Administración y no mediante el recurso al mandato.

Así, de una parte, deben tenerse en consideración las previsiones de la Ley 2/2011, de 5 de mayo, de Economía Sostenible sobre "Sostenibilidad Ambiental", que pretenden crear el marco jurídico del fomento de las energías renovables y la eficiencia energética y que, sin embargo, parecen más un código de buenas intenciones ${ }^{27}$ que una norma que estructure la ordenación de las energías renovables y la eficiencia energética. En este sentido, baste pensar en el art. 85 de la norma, que dispone que "todas las Administraciones, en el ejercicio de sus respectivas competencias, incorporarán los principios de ahorro y eficiencia energética".

Junto a ello, debemos destacar la planificación en materia de energías renovables y eficiencia energética, cuyo objetivo es conseguir la reducción de emisiones de GEI por la doble vía de incrementar el recurso a fuentes de energía renovables y reducir el consumo energético a través de dispositivos de ahorro y eficiencia energética ${ }^{28}$.

La peculiaridad de este escenario es la escasa atención que se le presta a los Entes Locales, sea porque no se desciende a especificar las competencias o acciones que deben emprender estas Administraciones, sea porque solo se precisan los objetivos que han de alcanzarse en un nivel general, y los Entes Locales aparecen como destinatarios de medidas y actuaciones que se deciden en un nivel superior. En consecuencia, y esto es lo que interesa, a efectos de conocer los instrumentos jurídicos de los que disponen las Administraciones Locales para lograr los objetivos de reducción de emisiones de GEI debe concretarse, en primer lugar, si el papel que juegan estos Entes es el de sujetos activos en la aplicación de medidas o, por el contrario, les corresponde ser destinatarios de las previsiones recogidas en los aludidos planes, asumiendo, así pues, un cierto rol ejemplarizante para los ciudadanos. Junto a ello, en un segundo momento habrá que conectar estas funciones con la capacidad, extensión e intensidad de actuación de los Entes Locales, para saber si su implicación en el ámbito de estudio de este Trabajo se desenvuelve en el diseño de las políticas ambientales, en la planificación o, finalmente,

Locales en la consecución de objetivos globales, por ejemplo a través de la participación en la Estrategia de desarrollo local sostenible mediante las Agendas Locales.

${ }^{27}$ Véase FERNÁNDEZ DE GATTA, “El régimen...”op. cit. pág.193.

${ }^{28}$ Véase Plan de Acción de Ahorro y EE 2011-2020, en relación con el art. 79 de la Ley de Economía Sostenible, que expresamente regula "la planificación energética indicativa", en clara contraposición con el art. 80 de la misma norma, relativo a la "planificación energética vinculante". 
en la intervención, sea a través de instrumentos de orden y control, sea a través de dispositivos de control indirecto, vinculados al fomento, la difusión de información o, incluso, la contratación pública ${ }^{29}$.

En todo caso, y como premisa del siguiente epígrafe, cualquiera que sea el nivel en el que nos situemos, lo cierto es que la implantación de las energías renovables y, sobre todo, la generalización de prácticas de eficiencia energética, ponen de manifiesto la complejidad de los instrumentos jurídicos que están utilizando las Administraciones Públicas, cuando el interés general se logra a través de la consecución de objetivos cuantificados numéricamente y compartidos por diversos niveles administrativos ${ }^{30}$, entre los que la cooperación y la coordinación, como principios de relación, pasan a un primer plano, planteándose la necesidad de combinar instrumentos de diversa naturaleza $^{31}$ que permitan la implicación activa de todas las Administraciones.

Finalmente, y como hemos señalado con anterioridad ${ }^{32}$, la sostenibilidad local en el ámbito de las energías renovables y la eficiencia energética también se nutre de un instrumento jurídico tan peculiar como el de los Planes de Acción, que se vinculan al llamado Pacto de los Alcaldes ${ }^{33}$, en cuya virtud la intervención de las Administraciones

${ }^{29}$ Sobre la aplicabilidad de la contratación del sector público como fórmula de tutela ambiental, a través de la inclusión de cláusulas de esta naturaleza en los pliegos de cláusulas técnicas particulares, véase, entre otros, PERNAS GARCÍA, J., Contratación Pública Verde, La Ley, Madrid, 2011, y FERNÁNDEZ DE GATTA, D., "La integración de aspectos ambientales en la contratación pública", en A. PIGRAU SOLÉ/L. CASADO CASADO (dirs.), Derecho Ambiental y transformaciones de la actividad de las administraciones públicas, Ed. Atelier, Barcelona, 2010.

${ }^{30}$ Véase Preámbulo Estrategia Europa 2020: Una Estrategia para un crecimiento inteligente, sostenible e integrador (Comunicación Comisión Europea, de 3 de marzo de 2010 (COM (2010): "el crecimiento sostenible se traduce en un incremento de las energías renovables y de un $20 \%$ de eficiencia energética [...]", lo cual implica el recurso a la reglamentación, las normas de la construcción e instrumentos de mercado tales como los impuestos, subvenciones y contratación pública "para reducir el consumo de energía y recursos y utilizar los fondos estructurales para intervenir la EE de los edificios públicos y en un reciclaje más eficaz" (pág. 17). En la misma línea, la Estrategia Española de Cambio Climático y Energía Limpia: Horizonte 2007-2012-2020 (Aprobado por el Consejo de Ministros el 2 de noviembre de 2007) diferencia entre políticas y medidas para mitigar el cambio climático y medidas para la consecución de consumos energéticos compatibles con el desarrollo sostenible, como la planificación en materia energética de las Administraciones. Lo destacable de estos documentos se encuentra en la ausencia de una consideración específica en lo que a las Administraciones Locales se refiere.

${ }^{31}$ En la Estrategia Española de Cambio Climático y Energía Limpia: Horizonte 2007-2012-2020, las acciones que se plantean van desde la realización de campañas de información hasta el estímulo de compromisos voluntarios con las empresas o el recurso a los mecanismos regulatorios.

${ }^{32}$ Véase supra nota al pie núm. 12.

${ }^{33}$ El objeto fundamental del Pacto es articular la posibilidad o competencia de los gobiernos locales y regionales de poner en marcha "medidas de eficiencia energética, proyectos de energías renovables y otras acciones relacionadas con la energía en diversas áreas de actividad de su competencia". Los Gobiernos Locales actúan, así, como "consumidor y proveedor de servicios" en cuestiones tan diversas 
que nos ocupan debería articularse a través de un Plan de Acción, con líneas estratégicas $^{34}$ que van desde la movilidad sostenible hasta la potenciación de las energías renovables e, incluso, la previsión de iniciativas tan especiales e inesperadas como la articulación de un mercado local de derechos de emisión para áreas geográficas especializadas en la reducción de emisiones. En este sentido, la actuación administrativa local quedaría concretada en cuestiones diversas como la participación de servicios municipales y organizaciones, la celebración de campañas de divulgación ${ }^{35}$ y promoción de la participación ciudadana o la realización de proyectos como los de peatonalización de los centros de las ciudades o la rehabilitación de edificios, teniendo en cuenta los objetivos de eficiencia energética.

A nuestro juicio, y sin perjuicio de lo que se dirá a continuación, la preeminencia en el sector que nos ocupa de Planes y Programas de Acción nos sitúa ante un Derecho administrativo blando que, consciente de las dificultades de compatibilizar las exigencias de la política energética con la ambiental de manera exclusiva, no apuesta por las energías renovables y la eficiencia energética a través de una regulación taxativa y determinante en la ordenación del sector ${ }^{36}$. Antes al contrario, en tanto en cuanto las Administraciones son sujetos activos de las medidas previstas en los aludidos planes, y estas medidas se sitúan en la esfera del fomento y la dirección indirecta, parece que la obligación mediante la imposición de objetivos numéricos, con discrecionalidad, por tanto, respecto de los medios que pueden utilizarse para su consecución, se convierte en la fórmula idónea para la creación del marco jurídico de las energías renovables y la eficiencia energética. Esto evidencia, por un lado, la especialidad del sector y, por otro, la complejidad creciente del Derecho administrativo, al que se van incorporando

como los edificios públicos, contratos de adquisición de bienes y servicios, planificación urbanística y organización del sistema de transporte, productor y proveedor de energía, etc.

${ }^{34}$ Esta es la conclusión fundamental del Documento de la FEMP "Análisis de los Compromisos derivados de la adhesión al Pacto de los Alcaldes", de 19 de noviembre de 2008, en cuya virtud se valoran las consecuencias de adhesión al referido Pacto.

35 Sobre esta cuestión, véase MOREU CARBONELL, E., La Administración Anunciante: régimen jurídico de la Publicidad Institucional, Ed. Thomson-Aranzadi, Cizur Menor (Navarra), 2005, págs. 204 y siguientes, en relación con el concepto de "publicidad institucional de promoción social", que, como señala la autora, puede tener entre sus fines la promoción de determinados valores o comportamientos sociales relacionados con la protección del medio ambiente.

${ }^{36}$ Sobre las dificultades de trasladar los instrumentos del soft law al Ordenamiento Jurídico a través del establecimiento de prescripciones y mandatos jurídicos "que permitan a sus destinatarios y a los operadores jurídicos tener una seguridad y certeza [...]", véase SANZ LARRUGA, "Sostenibilidad ambiental..." op. cit., pág. 438. 
instrumentos jurídicos diversos que concretan los objetivos y fines de la actuación administrativa.

Desde esta perspectiva, no hay duda de que la "intervención" administrativa adquiere una significación mayor de aquella que se vinculaba a la actividad de policía, para poder dar cobijo, así, a una multiplicidad de dispositivos jurídicos que no siempre pueden identificarse con la actividad de limitación. Las Administraciones públicas tienen nuevas funciones, y éstas precisan un instrumentario propio para el que, a nuestro juicio, las energías renovables y los objetivos de eficiencia energética constituyen un sector de referencia.

\section{DISPOSITIVOS DE ACTUACIÓN Y/O INTERVENCIÓN DE LOS ENTES LOCALES EN MATERIA DE ENERGÍAS RENOVABLES Y EFICIENCIA ENERGÉTICA}

Analizado el marco competencial y de actuación de los Entes Locales para intervenir en materia de energías renovables y eficiencia energética, y teniendo en cuenta las peculiaridades técnicas del sector, la consideración de los instrumentos jurídicos de los que pueden disponer estos entes precisa del reconocimiento de un doble plano para la sistematización de dichos instrumentos, en aras de la articulación y el establecimiento del medio urbano sostenible del que partíamos en este trabajo.

Así, en primer lugar, cabe atender a la posición que ocupan estos Entes en la generalización de las energías renovables y la reducción de consumos energéticos a través de la eficiencia energética; ello nos permite diferenciar entre la posibilidad de que los Ayuntamientos (y las Diputaciones, en su caso) puedan ser sujetos activos en la consecución de los objetivos aludidos y, por extensión, en el desenvolvimiento de la función pública de tutela ambiental, o, por el contrario, que estas Administraciones puedan ser meros destinatarios de las medidas previstas en los planes, como ocurre en la puesta en marcha de medidas "ejemplarizantes", o en la recepción de ayudas y subvenciones para ámbitos como el cambio de alumbrado público o la modernización de dispositivos técnicos. A nuestro juicio, cuando del análisis de las normas y planes aplicables al ámbito de las energías renovables y la eficiencia energética, esta última sea la posición de la entidad local, es evidente que no hay margen de maniobra para la misma en orden a la configuración de una política de sostenibilidad propia, puesto que 
se convierte en mero ejecutor de la medida prevista en un nivel superior.

En contraposición, cuando las Entidades Locales se sitúan en la aludida posición activa, la intervención o participación de las Administraciones Locales en materia de energía puede alcanzar distintos grados, atendiendo al carácter vinculante o no de la medida, y, por tanto, al alcance más o menos programático de la misma en este último caso, lo que ofrece una diversidad de instrumentos jurídicos que ponen de manifiesto las especialidades de ordenación de esta materia.

Desde esta perspectiva, un primer grado de la intervención de las Administraciones Locales consistiría en la participación en el diseño de políticas ambientales en la materia que tratamos y/o la implicación en la elaboración de una estrategia integral de desarrollo urbano sostenible. Como hemos visto, el escenario normativo y la planificación de instancias superiores dejan un espacio muy pequeño a la iniciativa local, pero no deben desdeñarse las posibilidades de participación activa que ofrece la puesta en marcha de las Agendas 21 o la adhesión al Pacto de los Alcaldes. En este caso, no hay duda de que los límites de la actuación administrativa se encuentran en los medios materiales y técnicos de los que disponga la entidad para concretar sus compromisos de sostenibilidad.

Por su parte, una de las actuaciones locales más extendida es la creación de entes instrumentales al modo de las Agencias provinciales o municipales de la energía como un ámbito de decisión de los Ayuntamientos y Diputaciones, que tienen, así, capacidad para articular una solución organizativa $a d$ hoc en la materia que nos ocupa, aunque, evidentemente, ligada a la potestad de autoorganización de estas Administraciones. En este sentido, son numerosos los ejemplos que existen en la mayoría de las Comunidades Autónomas, lo que evidencia la disparidad de entes creados en lo que respecta a su naturaleza jurídica y personalidad jurídica, así como en relación con las funciones que han de asumir ${ }^{37}$. No obstante esta última afirmación, puede reconocerse un cierto protagonismo de la actividad de fomento en estas Agencias, entendido en un sentido amplio y estrechamente relacionado con el impulso de programas e iniciativas para el logro de los aludidos objetivos cuantificados de generalización del recurso a las energías

\footnotetext{
${ }^{37} \mathrm{Al}$ respecto, véase la exposición sistemática que lleva a cabo LÓPEZ SAKO en "Competencias...” op. cit., págs. 488 a 491 .
} 
renovables y comportamientos o prácticas de eficiencia energética ${ }^{38}$.

En un segundo nivel de intervención, y teniendo en cuenta el grado de concreción y vinculatoriedad de los instrumentos jurídicos a los que recurren las Administraciones Locales, debemos considerar la actividad de planificación y las potestades que los Entes Locales tienen reconocidas al efecto, que hace que los planes se conviertan en un instrumento fundamental para la promoción de las energías procedentes de fuentes renovables en lo que respecta a la previsión de infraestructuras. Desde esta perspectiva, los planes urbanísticos son manifestación de una clara intervención en materia de energías renovables y eficiencia energética en aquellos supuestos en los que, a modo de ejemplo, se opta por el diseño de ciudades compactas, con la consiguiente reducción de consumo energético al ofrecer servicios básicos en la mayoría de núcleos urbanos dentro de municipio, o llevar a cabo un trazado determinado de las vías que favorezca los desplazamientos en transporte público o a pie $^{39}$.

Junto a ello, los Entes Locales, fundamentalmente en el ámbito municipal, se están también atreviendo a planificar de manera indicativa, mediante planes que, grosso modo, no se apoyan en un contenido de "ordeno y mando", porque los objetivos que el propio plan diseña pretenden exigirse de manera indirecta a través de la creación de un escenario a partir del cual el ciudadano pueda decidir. La cuestión no es baladí, porque

\footnotetext{
${ }^{38}$ Así, a modo de ejemplo y sin ánimo exhaustivo, en la Agencia Provincial de la Energía de Huelva, además de ofrecer información al ciudadano se están llevando a cabo en la actualidad dos programas: WICO (Wind of the coast), junto a dos localidades de Reino Unido e Italia, en el marco del Proyecto POWER, dependiente del Programa Europeo de Cooperación Territorial INTERREG IVC, para evaluar la potencialidad de la energía eólica en zonas próximas a la costa, principalmente introduciendo criterios de planificación y búsqueda de las barreras existentes para el uso de la energía mini-eólica; se prevé el intercambio de Buenas Prácticas. El otro Proyecto se denomina RURAL-RES, en el que, contando como socios a Suecia, Italia, Grecia, Rumanía y la República Checa, se quiere promover la producción de energías renovables a través del desarrollo de plantas mini-hidraúlicas y mini-eólicas conectadas a red, e instalaciones mini-eólicas aisladas, a fin de demostrar que estas instalaciones son compatibles con los espacios naturales y otras actividades como el turismo; entre los resultados del Proyecto está la publicación de dos Guías sobre "Buenas Prácticas en plantas mini-hidráulicas" y "Buenas Prácticas en instalaciones mini-eólicas", que tienen la relevancia de acercarnos a un Derecho administrativo de carácter "blando".

${ }^{39}$ En este sentido, el art. 3.d) de la Ley 2/2007, de 27 de marzo, de Fomento de las Energías Renovables y el Ahorro Energético de Andalucía, contempla como principio inspirador de la misma "la articulación territorial del sistema energético en base a una generación distribuida que suponga una minimización de las pérdidas en el transporte y distribución de la energía". Por su parte, podemos destacar, sin ánimo exhaustivo, la previsión del art. 11.3 de la misma Ley, al contemplar la posibilidad de que los planes urbanísticos establezcan "áreas preferentes" a fin de garantizar espacios vinculados a la generación y transporte de energía procedente de fuentes renovables. Sobre una consideración más detenida de las previsiones del Derecho Autonómico, véase MORA RUIZ, M., "La ordenación jurídico-administrativa de las energías renovables en nuestro Derecho: Justificación, características y evolución de un sector esencial en la lucha contra el cambio climático", en Noticias de la Unión Europea, en prensa, págs. 24 y siguientes.
} 
pone de manifiesto que la Administración considera que, en determinados ámbitos de actuación, y dado que implican un cambio en la percepción social del mismo, no cabe sino la recomendación, la información o el fomento cuando las medidas se dirigen a los ciudadanos, sin perjuicio de que sí estemos ante una planificación vinculante para las Administraciones destinatarias del mismo, en el sentido de que concreta sus objetivos, plazo de ejecución y acciones para el logro de tales objetivos. A nuestro juicio, éste es el tipo de planificación característico en las energías renovables y la eficiencia energética, de tal forma que los Entes Locales no hacen sino imitar a las Administraciones estatal y autonómica mediante la aprobación de estos planes y, en el ámbito de sus propios intereses, creando un cierto espacio en el que las energías renovables cuentan con instrumentos propios que, de otra manera, no serían sino aspectos parciales de otras políticas públicas con más solvencia, como el medio ambiente o el urbanismo.

No obstante lo anterior, este tipo de planes no se ha aprobado con carácter general, y son pocos los ejemplos que se pueden examinar. En este sentido, del análisis de dos de estos instrumentos - el Plan Municipal de Ahorro y Eficiencia Energética y Energías Renovables de Miguelturra, en Ciudad Real ${ }^{40}$, y el Plan de Acción para el Ahorro de Energía y Reducción de las Emisiones a la Atmósfera de Barcelona ${ }^{41}$ — pueden destacarse los siguientes aspectos:

En primer lugar, el Plan puede concebirse como un plan para la gestión del Ayuntamiento dentro de un Plan de Acción más amplio e integral, en el marco de la Agenda 21, y teniendo como objetivo la lucha contra el cambio climático desde el ámbito local —es el enfoque del aludido Plan de Miguelturra—. Así, es posible la identificación de instrumentos diversos, que presentan, a su vez, distinta naturaleza técnicos, como las auditorías; organizativos, económicos, divulgativo-informativos y participativos-, de tal forma que las acciones realizadas han consistido, en el Plan examinado, en la instalación en edificios públicos de sistemas solares térmicos y fotovoltaicos, la renovación del alumbrado público y la conversión de la Administración local en un referente en la aplicación de "buenas prácticas energéticas para que sirva de modelo y ayude a desarrollar una economía de $\mathrm{I}+\mathrm{D}+\mathrm{i}$ en el municipio.

\footnotetext{
${ }^{40}$ Puede consultarse en $<$ www.ecourbano.es $>$, visitada el 8 de noviembre de 2011.

${ }^{41}$ El documento se encuentra accesible en $<$ www.barcelonaenergia.com $>$, visitada el 8 de noviembre de 2011.
} 
Por su parte, el Plan de Acción para el Ahorro de la Energía y Reducción de Emisiones a la Atmósfera de Barcelona resulta de interés porque se configura como un Plan de Acción a través del cual se diseña "una estratégica energética en el ámbito de Barcelona", con identificación de objetivos, asignación de recursos y gestión y seguimiento de los resultados. Así, el plan contempla diversas líneas estratégicas y, por tanto, prevé diferentes medidas en atención al sector en el que nos movamos: a modo de ejemplo, el sector residencial constituye un ámbito de proyectos y "recomendaciones" (en los términos del propio Plan), en el que se entiende que debe procederse a cuestiones tan diversas como la "mejora de carpintería y cristales de las ventanas", el fomento de las buenas prácticas y concienciación de los ciudadanos en lo que al alumbrado de bajo consumo en viviendas se refiere, o la revisión de "los estándares energéticos en la construcción de obra nueva y rehabilitación del interior de viviendas", a través de recomendaciones u ordenanzas. También son ámbitos de actuación los edificios e instituciones públicas, el sector servicios y comercial o el de oficinas. Pero, sin duda, el sector de mayor relevancia en lo que a la intervención administrativa se refiere es el llamado "Sector General", para el que se recogen propuestas e instrumentos jurídicos de distinta naturaleza que, por un lado, evidencian las dificultades de ordenación de este sector, y, por otro, ponen el acento en el papel activo que debe corresponder a los ciudadanos en la consecución de objetivos ambientales, en el marco de un principio de responsabilidad compartida en la gestión ambiental ${ }^{42}$. Así, se contemplan soluciones organizativas al modo de la Agencia de la Energía de Barcelona, la creación del Observatorio de la Energía, junto al impulso de un pacto de energía con agentes sociales y económicos, la creación de una "marca de calidad sobre la gestión y el uso de la energía en Barcelona", o la promoción de auditorías energéticas de carácter voluntario en las empresas y la aplicación de medidas de ahorro. A nuestro juicio, resulta evidente que estos dispositivos suponen una opción de la Administración municipal por el incentivo y por la acreditación de determinados comportamientos en materia de energías renovables y eficiencia energética, pasando a un segundo plano la ordenación consistente en la intervención directa de las Administraciones. Ésta parece ser la solución ordenadora más lógica en el ámbito en el que nos movemos, pues, en última instancia, las energías renovables y la eficiencia energética son sólo un aspecto

\footnotetext{
${ }^{42}$ Véase MORA RUIZ, M., La gestión ambiental compartida: función pública y mercado, Ed. Lex Nova, Valladolid, 2007, in totum.
} 
parcial de una política energética de mayor envergadura que sí dispone de una regulación en un sentido más estricto del término.

Finalmente, en el que podría considerarse el nivel más alto en cuanto a la intensidad de la intervención de las Administraciones, los Entes Locales disponen de dispositivos de orden y control para el logro de los objetivos a los que venimos haciendo referencia; en concreto, pueden valerse de autorizaciones, licencias y regulaciones restrictivas como las que representan las Ordenanzas relativas a la captación de energía solar ${ }^{43}$. Así, tomando como referencia la Ordenanza de 5 de noviembre de 2008 de Jaén sobre Captación y Aprovechamiento de Energía Solar Térmica en Edificios ${ }^{44}$, en tanto que regulación bastante completa, cabe destacar que los Ayuntamientos pueden valerse de licencias para exigir la incorporación de sistemas de captación y utilización de energía solar para usos como la producción de agua caliente sanitaria, piscinas, etc. Lo llamativo de la regulación es que se convierte en exigencia para obras públicas y privadas, así como para las empresas de instalación, en el caso de que deban obtener licencia de obras, ocupación o actividad y funcionamiento, así como licencias equivalentes $^{45}$ (arts. 2 y 3), con clara limitación para la libertad de empresa; se crea, así pues, un espacio para la actividad de limitación.

En este sentido, el modelo previsto por la Ordenanza apunta claramente a la intervención en el ámbito al que se refiere, tal y como ponen de manifiesto los arts. $10 \mathrm{a}$ 12 de la norma, al referirse, respectivamente, a la potestad de inspección de servicios (por técnicos del Ayuntamiento y empresas autorizadas), la revisión de licencias por la Gerencia Municipal de Urbanismo y el correspondiente catálogo de infracciones y sanciones ${ }^{46}$. No obstante, la intervención queda atenuada por dos cuestiones de distinta

\footnotetext{
${ }^{43}$ En el siguiente enlace puede encontrarse referencia de las Ordenanzas municipales sobre energía aprobadas más recientemente: <http://www.vidasostenible.org/local/local2.asp?id=156>, visitada el 18 de febrero de 2012.

${ }^{44}$ BOP núm. 282, de 9 de diciembre; rect. BOP núm. 48, de 27 de febrero de 2009. En este ámbito, debe destacarse la Propuesta del Modelo de Ordenanza Municipal para la captación de energía solar para usos térmicos llevada a cabo por los profesores J. ORTEGA BERNARDO y A. GALLEGO ANABITARTE, accesible

$<$ http://www.idae.es/index.php/mod.documentos/mem.descarga?file=/documentos 5637 OrdenanzaSolar UsosTermicos_2002_47b2e32f.pdf>, visitada el 18 de febrero de 2012.

${ }^{45}$ En este sentido, debe recordarse que el art.84.bis LRBRL ha eliminado, en aras de la simplificación y la liberalización de servicios, las licencias de actividad salvo que haya cuestiones ambientales que legitimen su exigencia, como sucede en el caso de la implantación de instalaciones o sistemas relativos a las energías renovables.

46 En esta línea se encontraría, también, la Ordenanza General de Medio Ambiente Urbano del Ayuntamiento de Barcelona en lo que a la Captación Solar se refiere, tal y como se pone de manifiesto en
} 
envergadura que, de nuevo, ponen de manifiesto las dificultades de un modelo clásico de actividad administrativa en el ámbito que nos ocupa, y que plantean una cierta incertidumbre sobre el contenido de la ordenación administrativa de las energías renovables y la eficiencia energética, también en el ámbito local. Así, debemos hacer referencia, de manera específica, al art. 13, en cuya virtud se contempla una línea de ayuda para la aplicación de la Ordenanza, y a la Disposición Transitoria, que de manera al menos llamativa señala que la "Ordenanza tiene carácter de recomendación", con el efecto fundamental, a nuestro juicio, de convertir a la Ordenanza en soft law, lo cual no sólo es un dato más que redunda en las peculiaridades del sector ${ }^{47}$, sino también a la hora de insistir en la necesidad de actualizar de manera considerable parte de los instrumentos jurídicos a través de los cuales se ha articulado la actividad administrativa con carácter general.

La consecuencia de lo afirmado en el párrafo anterior es, para nosotros, la vinculación del sector de las energías renovables y la eficiencia energética a la que podríamos considerar actividad de dirección indirecta de las Administraciones, lo que lo conecta con la amplia diversidad de dispositivos que dichas Administraciones utilizan para conseguir el objetivo de generalizar el recurso a fuentes de energías renovables y el ahorro energético. Desde esta perspectiva, debe pensarse que la Directiva de fomento de las energías renovables se basa en la determinación, por parte de los Estados miembro de la Unión Europea (UE), del sistema de apoyo que consideren oportuno para el logro de los objetivos cuantificados de la norma, lo cual no siempre puede identificarse con la actividad de fomento en términos más o menos tradicionales, sin perjuicio de que a éste corresponda un lugar privilegiado entre los sistemas de apoyo ${ }^{48}$. Los Entes Locales proyectan este enfoque y otorgan un papel principal a aquellas medidas características del fomento, como el otorgamiento de subvenciones y ayudas, la valoración de criterios de eficiencia energética o la aplicación de energías renovables en la contratación pública, pero, como hemos visto, esto no desplaza por completo a las técnicas jurídicas propias de la actividad de limitación. En todo caso, y pese al papel limitado de las Entidades Locales en la generalización de las energías renovables y el ahorro

\footnotetext{
los arts. 4 a 7 y 17 a 20 (BOP núm. 181, de 30 de julio de 1999; correc. BOP núm. 265, de 5 de noviembre de 1999).

${ }^{47}$ Véase MORA RUIZ, “La ordenación...” op. cit., págs. 193, 194.

${ }^{48}$ Sobre esta línea argumental, véase MORA RUIZ, “Los condicionantes...”, op. cit., págs. 26 a 30.
} 
energético, estas Administraciones se sitúan, en el espacio de competencia que le es propio, en la misma línea de acción que el resto de las Administraciones.

Desde esta perspectiva, y con ello concluimos el presente epígrafe, la dirección indirecta a la que nos referíamos supone también una implicación mayor y más activa del ciudadano en el logro de objetivo ambientales, de tal forma que la información y formación que las Administraciones han de proporcionar a la sociedad para cambiar los hábitos vitales se convierte en una pieza clave de las nuevas funciones que han de corresponder a las Administraciones, destacando muy especialmente el rol ejemplarizante que las Entidades Locales asumen, en los términos en que se ha expuesto. Cómo se gestiona esta información constituye, sin duda, otra de las líneas de evolución que las energías renovables y la eficiencia energética pueden plantear al Derecho administrativo.

\section{CONSIDERACIONES FINALES}

A la vista de todo lo expuesto, este Trabajo debe concluir con algunas consideraciones sobre el papel, competencias y actividades que pueden asumir las Entidades Locales en materia de energías renovables y ahorro y eficiencia energética, desde un punto de vista inicial puramente ambientalista, en cuya virtud las exigencias del principio de desarrollo sostenible han de satisfacerse, entre otras cuestiones, mediante la generalización del recurso a la energía procedente de fuentes renovables, así como del ahorro y la eficiencia energética, para lograr el objetivo ambiental de reducción de las emisiones de GEI. En este sentido, la concreta ordenación de este sector, a la luz de la Directiva 2009/28/CE, del Parlamento y del Consejo, de 23 de abril, de fomento de las energías renovables, plantea un escenario jurídico novedoso, en el que es posible alternar o combinar los instrumentos considerados de orden y control para atender a las exigencias de la tutela ambiental con otros dispositivos de dirección indirecta, menos incisivos para el ejercicio de derechos como la libertad de empresa, lo que favorece que el sector que nos ocupa conecte con la línea de evolución reconocida del Derecho administrativo ambiental consistente en "la ampliación del elenco de instrumentos orientados a la protección ambiental" ${ }^{\prime 9}$ sobre la base de una necesaria colaboración entre sujetos

\footnotetext{
${ }^{49}$ Esta es la valoración, que compartimos, que realiza BETANCOR, A., en "Nuevas tendencias del Derecho Ambiental. Perspectiva general introductoria", en Revista General de Derecho Administrativo, núm. 25, 2010, pág. 15 .
} 
públicos y privados para lograr el objetivo común de reducción de las emisiones de GEI. Desde esta perspectiva, las consideraciones realizadas en este Trabajo nos permiten afirmar que la regulación de estas energías renovables y la eficiencia energética pueden situarse en la vanguardia de los dispositivos de tutela ambiental, y de ahí el interés por concretar el grado de participación y el instrumentario jurídico que las Entidades Locales pueden ofrecer para el desenvolvimiento de la función pública de protección del medio ambiente.

En este sentido, sin embargo, hemos de concluir que dicha participación activa de todas las Administraciones en este sector no ha producido el efecto de creación y configuración de un ámbito de actuación propio de las Administraciones Locales, con la correspondiente atribución de competencias. Antes bien, pese a la existencia de previsiones generales para todas las Administraciones, lo cierto es que las posibilidades de acción de los Entes Locales no se han apoyado en la legislación y planes aprobados en la materia que nos ocupa, pasando a un primer plano la legitimación que les otorga la noción de sostenibilidad y el impulso internacional de la acción local en los términos que se han señalado. En nuestra opinión, esta última afirmación supone que la función pública de tutela ambiental local a la que acabamos de referirnos dependerá, en gran medida, no tanto del marco jurídico en el que poder desenvolverse como de los medios materiales y económicos de los que dispongan las Entidades Locales para hacer efectiva una determinada política ambiental. La cuestión, a nuestro juicio, no es baladí, ya que la articulación de la ordenación jurídica de las energías renovables y la eficiencia energética constituye un aspecto fundamental para la generalización del sector, que, de ese modo, podría articularse de manera racional.

No obstante lo anterior, no puede desconocerse el esfuerzo de los Entes Locales en, por un lado, configurar una política propia de generalización del recurso a las energías procedentes de fuentes renovables y la consecución de objetivos de ahorro energético, en el marco de sus propios intereses, y, por otro, en recurrir no sólo a los instrumentos jurídicos propios o característicos del ejercicio de sus potestades, sino también, en claro paralelismo con la Administración estatal o las autonómicas, en utilizar otros dispositivos alternativos a los de la intervención directa sobre la esfera de derechos de los ciudadanos; tal cosa refleja sin duda la variedad de instrumentos jurídicos, en cuanto a su naturaleza, que interactúan en el ámbito que nos ocupa. 
A nuestro juicio, y con ello concluimos, estas valoraciones ponen de manifiesto las dificultades de ordenación del sector que ya hemos señalado, por lo que debe cambiarse el enfoque en la consideración o el estudio del mismo, pues lo realmente interesante no reside en la visión tradicional de qué es lo que puede o lo que no puede hacer la Administración frente al particular, sino en cómo la Administración regula el sector y, por tanto, cómo construye el mismo en positivo. Los Entes Locales no pueden ser ajenos a este proceso de construcción, tal y como se demuestra a través de los instrumentos de actuación y/o intervención considerados, por lo que el marco jurídico de las energías renovables y la eficiencia energética debe permitir un espacio necesario para que dichos Entes efectúen el diseño de su política energética, ajustada a su ámbito de actuación. Desde esta perspectiva, la clave no está en determinar las competencias legitimadoras de la acción local en energías renovables, sino en intensificar la cooperación de las Administraciones Públicas y, en menor medida, la coordinación, ya que la sostenibilidad urbana otorga a los Entes Locales el marco jurídico necesario para tomar sus propias decisiones y contribuir a un objetivo global de lucha contra el cambio climático.

\section{BIBLIOGRAFÍA CITADA}

ALONSO GARCÍA, E., en "La gestión del medio ambiente por las Entidades Locales", en S. MUÑOZ MACHADO, (Dir.), Tratado de Derecho Municipal, Tomo II, Iustel, Madrid, 2011.

BETARNCOR, A., "Nuevas tendencias del Derecho Ambiental. Perspectiva general introductoria", en Revista General de Derecho Administrativo, núm. 25, 2010.

CANALS I AMETLLER, D., "Las Administraciones en el cumplimiento del Protocolo de Kioto. El mercado de derechos de emisión de gases de efecto invernadero y otros instrumentos", en J. ESTEVE PARDO, (Dir.), Derecho del Medio Ambiente y Administración Local, 2a Edición, Fundación Democracia y Gobierno Local, Barcelona, 2006.

FERNÁNDEZ DE GATTA, D., "El régimen de sostenibilidad medioambiental", Revista Jurídica de Castilla y León, 25 septiembre, 2011. 
— "La integración de aspectos ambientales en la contratación pública", en A. PIGRAU SOLÉ/L. CASADO CASADO (Dirs), Derecho Ambiental y transformaciones de la actividad de las administraciones públicas, Ed. Atelier, Barcelona, 2010.

GONZÁLEZ RÍOS, I., Régimen jurídico-administrativo de las energías renovables y de la eficiencia energética, Ed. Aranzadi, Cizur-Menor, Navarra, 2011.

LÓPEZ SAKO, M.J., "Competencias municipales en materia de energías renovables", en D. VERA JURADO (Coor.), Competencias ambientales de las Entidades Locales de Andalucía, CEMCI, Granada, 2009.

- Regulación y Autorización de los parques eólicos, Thomson-Civitas, Madrid, 2008 .

LORA-TAMAYO, M., "La integración normativa de la dimensión medioambiental en el medio urbano. El desarrollo urbano sostenible y su implementación", en R. PARADA VÁZQUEZ, A FUENTETAJA PASTOR, Reforma y retos de la Administración local, Ed. Marcial Pons, Madrid, 2007.

MORA RUIZ, M., "La ordenación jurídico-administrativa de las energías renovables en nuestro Derecho: Justificación, características y evolución de un sector esencial en la lucha contra el cambio climático", en Noticias de la Unión Europea, en prensa.

— "La ordenación jurídico-administrativa de las energías renovables: revisión en el marco de la Directiva 2009/28/CE, del Parlamento Europeo y del Consejo, de 23 de abril, de fomento de las energías renovables, en Revista de Derecho Urbanístico y Medio Ambiente, núm. 257, 2010.

— "Los condicionantes ambientales de las energías renovables: el ejemplo de la energía eólica en el Derecho Español”, en Actualidad Jurídica Ambiental, 30 de septiembre de 2010 (en <www.actualidadjuridicaambiental.com>).

- La gestión ambiental compartida: función pública y mercado, Ed. Lex Nova, Valladolid, 2007.

MOREU CARBONELL, E., La Administración Anunciante: régimen jurídico de la Publicidad Institucional, Ed. Thomson-Aranzadi, Cizur Menor (Navarra), 2005. PERNAS GARCÍA, J., Contratación Pública Verde, La Ley, Madrid, 2011. 
SANZ LARRUGA, J., "Sostenibilidad ambiental y Derecho Administrativo: ¿nuevo remedio ante la crisis económica o una exigencia constitucional? A propósito de la nueva Ley de Economía Sostenible", en A. BLASCO ESTEVE (Coor.), Actas del VI Congreso de la Asociación Española de Profesores de Derecho Administrativo, INAP, Madrid, 2011. 